Journal of Case Reports 2017;7(3):267-269

\title{
Neonatal Meningitis with Unusual Bug?
}

\author{
Hadi M Fakih ${ }^{1,2}$, Farah Daakour ${ }^{1}$ \\ ${ }^{1}$ Department of Pediatrics, Faculty of Medical Sciences, Lebanese University, Beirut, Lebanon; ${ }^{2}$ Department of Pediatrics, NICU \\ Division, Sheikh Ragheb Harb Hospital, Toul, Lebanon.
}

\section{Corresponding Author: \\ Dr. Hadi M Fakih \\ Email: hadifakih75@gmail.com}

This is an Open Access article distributed under the terms of the Creative Commons Attribution License (creativecommons.org/ licenses/by/3.0).

Received Accepted Published

\begin{abstract}
Background: Neonatal meningitis remains a life threatening invasive infection that needs a comprehensive approach. Early diagnosis and institution of appropriate empiric antibiotic therapy will be life-saving and decrease complications. Case Report: We report the case of a fifteen day-old boy presenting for sepsis evaluation with one day history of high grade fever, irritability and poor feeding. Sepsis work up revealed meningitis secondary to Moraxella catarrhalis. Conclusion: Moraxella catarrhalis is a gram negative bacteria that is rarely reported in late onset neonatal sepsis, and we will review the latest literature on neonatal meningitis secondary to M. catarrhalis.
\end{abstract}

Keywords: Infant, Meningitis, Moraxella catarrhalis, Neonatal Sepsis, Newborn.

\section{Introduction}

Neonatal bacterial meningitis continues to be an important cause of mortality and morbidity. The mortality rates attributable to neonatal meningitis are substantial (5\%-25\%), and approximately $25 \%$ to $50 \%$ of the survivors sustain neurologic sequelae, including cognitive impairment and developmental delay [1]. Despite the development of effective vaccines, a useful tool for rapid identification of pathogens and potent antimicrobial drugs, neonatal meningitis continues to contribute substantially to neurological disability worldwide. An early empiric antibiotic treatment is critical for the management of neonates with bacterial meningitis, but early recognition of bacterial meningitis continues to be a challenge in neonates.

\section{Case Report}

A fifteen day of life male infant was admitted to our neonatal intensive care unit (NICU) for irritability, high grade fever and refusal to feed for last 24 hours. He was born by cesarean section from a 28 year old lady, gravida three, with smooth antepartum course of pregnancy. There was no vomiting, diarrhea or respiratory tract infection symptoms or skin rash. On admission to our unit, a full sepsis workup was performed given the stable hemodynamic status of the baby with no signs of organs dysfunction. The physical examination showed normal anterior fontanelle without any bulging.

Laboratory investigation revealed a leukocytosis up to 17,400 cells/ mL, with neutrophils predominance, a slightly increased C-reactive protein (CRP) to $22 \mathrm{mg} / \mathrm{dL}$ (normal value less than $10 \mathrm{mg} / \mathrm{dL}$ ), urine analysis was normal, chest X-ray showed no abnormality, the cerebrospinal fluid (CSF) studies revealed pleocytosis up to 370 cells/mL which was considered positive despite being a little hemorrhagic with red blood cells count in CSF up to 70,000 cells/mL. At this time empiric antibiotics treatment with ampicillin and ceftazidime was initiated pending the results of blood and CSF cultures that unfortunately turned out to be both negative. Polymerase chain reaction (PCR) test was also performed and uncovered the causative germ to be Moraxella catarrhalis. The baby completed a course of twenty one day of intravenous ceftazidime, without in hospital 
complications. The CT scan of brain was normal as was also the hearing test.

\section{Discussion}

Moraxella (Branhamella) catarrhalis, formerly called Neisseria catarrhalis or Micrococcus catarrhalis, is a gram-negative, aerobic diplococcus frequently found as a commensal of the upper respiratory tract. Moraxella catarrhalis has been reported with increasing frequency to be the etiological agent of serious systemic infection in both children and adults [2]. In immunocompromised hosts, the bacterium can cause a variety of severe infections including pneumonia, endocarditis, septicemia, and meningitis [3]. Bacteremia due to $M$. catarrhalis should be considered especially in febrile children with an underlying immune dysfunction and an upper respiratory tract infection [4]. Apart from its almost universal $\beta$-lactamase-mediated resistance to penicillins and its inherent resistance to trimethoprim, M. catarrhalis remains universally sensitive to most antibiotics used in the treatment of respiratory infections [5]. As in our case that had a complete resolution of symptoms without neurologic sequels after receiving the full course of intravenous ceftazidime.

Neonates with bacterial meningitis, however, usually present with subtle, variable, or non-specific features. These features might include fever, hypothermia, lethargy, poor feeding, irritability, vomiting, diarrhea, respiratory distress, seizures, or bulging fontanelles. Cerebrospinal fluid (CSF) examination is essential to establish the diagnosis of bacterial meningitis and to identify the causative organism and undertake in vitro antibiotic susceptibility testing. CSF culture is the gold standard for diagnosis of meningitis [1]. Characteristic CSF findings for acute bacterial meningitis are polymorphonuclear pleocytosis, hypo-glycorrhachia, and increased protein concentrations, but similar CSF findings can be demonstrated for neonates with intra-ventricular hemorrhage. The use of standard or sequential- multiplex polymerase chain reaction has been useful in identification of infecting bacteria. Detection of bacterial nucleic acid in CSF is most promising if Gram stain and culture of CSF results are negative because of preceding antibiotic therapy [1].

Treatment of neonates younger than 1 month at risk of early- and late-onset meningitis must include a combination regimen containing ampicillin and an aminoglycoside to cover Streptococcus agalactiae (GBS), E. coli, and L. monocytogenes, whereas a regimen including a third-generation cephalosporin is preferred for a case with a high likelihood of gram-negative bacilli. This empiric regimen is usually continued until the information is available on the infecting organism and antibiotic susceptibility. The duration of antibiotic therapy depends on the causative pathogen and its antimicrobial susceptibility and disease severity. A long-standing recommendation is usually 21 days of appropriate antibiotics for neonatal pathogens [1].

\section{Conclusion}

Neonatal meningitis remains a life threatening invasive infection that needs a comprehensive approach. Early diagnosis and institution of appropriate empiric antibiotic therapy will be lifesaving and reduce later complications. Molecular diagnostic approach should be routinely ordered to make the diagnosis of poorly cultured rare bacterium like Moraxella catarrahlis possible and guide our management.

Contributors: HAF: manuscript writing, literature search and case management; FD: manuscript editing, literature search and case management. HAF will act as guarantor. All authors approved the final version of the manuscript.

Funding: None; Competing interests: None stated.

\section{References}

1. Kwang SK. Neonatal Bacterial Meningitis. Neo Reviews. 2015;16:e535-e543.

2. Johnson MA, Drew WL, Roberts M. Branhamella (Neisseria) catarrhalis a lower respiratory tract pathogen? J Clin Microbiol. 1981;13:1066-1069. 
3. Daoud A, Abuekteish F, Masaadeh H. Neonatal meningitis due to Moraxella catarrhalis and review of the literature. Ann Trop Paediatr. 1996;16:199-201.

4. Abuhammour WM, Abdel-Haq NM, Asmar BI, Dajani AS. Moraxella catarrhalis bacteremia: a 10-year experience. South Med J. 1999 ;92:1071-1074.

5. McGregor K, Chang BJ, Mee BJ, Riley TV. Moraxella catarrhalis: clinical significance, antimicrobial susceptibility and BRO beta-lactamases. Eur J Clin Microbiol Infect Dis. 1998;17:219-234. 